\title{
Moxibustion Activates Macrophage Autophagy and Protects Experimental Mice against Bacterial Infection
}

\author{
Xiaojuan Li, ${ }^{1,2}$ Guanhua Guo, ${ }^{1,3}$ Feng Shen, ${ }^{1}$ Lihong Kong, \\ Fengxia Liang, ${ }^{1,2}$ and Guojie Sun ${ }^{1,2}$ \\ ${ }^{1}$ Acupuncture and Moxibustion College, Hubei University of Chinese Medicine, Wuhan, Hubei 430061, China \\ ${ }^{2}$ Hubei Provincial Collaborative Innovation Center of Preventive Treatment by Acupuncture and Moxibustion, \\ Wuhan, Hubei 430061, China \\ ${ }^{3}$ Shanxi University of Traditional Chinese Medicine, Taiyuan, Shanxi 030024, China
}

Correspondence should be addressed to Fengxia Liang; 315938821@qq.com and Guojie Sun; sunguojie@sina.com

Received 11 May 2014; Accepted 8 July 2014; Published 23 July 2014

Academic Editor: Gerhard Litscher

Copyright (C) 2014 Xiaojuan Li et al. This is an open access article distributed under the Creative Commons Attribution License, which permits unrestricted use, distribution, and reproduction in any medium, provided the original work is properly cited.

\begin{abstract}
Moxibustion is one of main therapies in traditional Chinese medicine and uses heat stimulation on the body surface from the burning of moxa to release pain or treat diseases. Emerging studies have shown that moxibustion can generate therapeutic effects by activating a series of signaling pathways and neuroendocrine-immune activities. Here we show moxibustion promoted profound macrophage autophagy in experimental Kunming mice, with reduced Akt phosphorylation and activated eIF2 $\alpha$ phosphorylation. Consequently, moxibustion promoted bacterial clearance by macrophages and protected mice from mortality due to bacterial infection. These results indicate that moxibustion generates a protective response by activating autophagy against bacterial infections.
\end{abstract}

\section{Introduction}

Autophagy is an evolutionary conserved physiological process by which protein aggregates and damaged organelles inside cells are selectively engulfed and delivered to and degraded in lysosomes $[1,2]$. This "self-eating" process is tightly associated with diverse physiological conditions including development and innate defense $[3,4]$ and a number of pathological conditions and diseases including cancers, neurodegenerative disease, and infectious diseases [5-7]. Emerging studies have shown that modulation of autophagy acts as a potential therapeutic strategy for treatment of disease $[8,9]$, even though autophagy is a double-edged sword in the progression of cancer and certain metabolic diseases [10-12]. Autophagy may restrict bacterial replication in the cytoplasm by clearance or be hijacked to support bacterial replication and survival $[13,14]$, a unique evasion technique of bacteria, which uses a variety of machinery to evade host defense systems to facilitate survival inside cells [15]. Autophagy primarily acts as an innate defense against bacterial infection [16], and enhanced autophagy provides a protective response against bacterial and virus infection $[17,18]$. However, it is difficult to modulate autophagy in unique lesions in vivo, which limits the application of autophagy as a therapeutic strategy.

Moxibustion is a physical therapy in traditional Chinese medicine that uses heat from the burning of minced herb moxa to stimulate acupuncture points for pain relief or disease treatment [19-21]. Moxibustion can affect neuroendocrineimmune functions and activate self-healing [22, 23]. Although the substitution of moxibustion by thermal physical therapy remains equivocal $[23,24]$, they both share the similar mechanism in self-healing and pain reduction [25, 26]. Emerging studies have shown that moxibustion alters the profile of gene expression, including cytokines [27-30] and signaling pathways [31-33], indicating it might be beneficial to patients with autoimmune and inflammatory diseases.

Previously, a protective response of moxibustion against HSV-1 infection was demonstrated in experimental mice [34]. However, the mechanisms of how moxibustion might be useful against infectious diseases remain to be fully defined. The present study shows that moxibustion activates 
autophagy of intraperitoneal macrophages in experimental Kunming mice, following the inactivation of Akt and the induction of eIF $2 \alpha$ phosphorylation. Consequently, it contributes to a protective response against bacterial infection, indicating moxibustion activates innate defenses to prevent infectious disease.

\section{Methods and Materials}

2.1. Mice and Ethics Statement. Specific-pathogen-free male Kunming mice (6-8 weeks of age, $18-22 \mathrm{~g}$ weight) were maintained and bred according to the guidelines approved by the Laboratory Animal Center, Sun Yat-sen University.

2.2. Mild Moxibustion on Lethal Infections of Staphylococcus aureus. Forty mice were randomly divided into four groups, A, control group; B, model group; C, 15 min moxibustion group; and D, 30 min moxibustion group, with 10 mice per group. Except for the control group, the remaining 30 mice were primed with intraperitoneal injection of $6 \%$ starch broth for 2 days, followed by intraperitoneal injection of 10 $\mathrm{LD}_{50}$ Staphylococcus aureus as an acute infection model. One hour after injection with bacteria, mice in groups $\mathrm{C}$ and $\mathrm{D}$ were restrained and administered $\phi 4 \mathrm{~mm}$ stick moxibustion for $15 \mathrm{~min}$ and $30 \mathrm{~min}$ at Guanyuan acupuncture point (CV 4 ) at the front of the small intestine. In the control group and model group, $\phi 4 \mathrm{~mm}$ sticks of unlighted moxa herbs were used as the control for moxibustion. The death of mice was monitored continuously for $48 \mathrm{~h}$ after injection to prepare a survival curve. Peritoneal fluids were collected immediately upon death or sacrifice at $48 \mathrm{~h}$ after moxibustion and subjected to bacterial colony formation inLuria-Bertani (LB) agar plates. The numbers of colonies were used to evaluate bacterial clearance.

2.3. Mild Moxibustion on Macrophage Autophagy. Forty mice were randomly divided into four groups: A, control group; B, model group; C, 15 min moxibustion group; and D, 30 min moxibustion group. In $\mathrm{B}, \mathrm{C}$, and $\mathrm{D}$ groups, mice received intraperitoneal injection of $6 \%$ starch broth as a macrophage inducer, and two days later mice in $\mathrm{C}$ and $\mathrm{D}$ groups were administered moxibustion at CV4 as described above and sacrificed at $1 \mathrm{~h}$ after moxibustion. Peritoneal fluid cells were collected and subjected to autophagy assays. To detect the dynamic Akt and eIF2 $\alpha$ phosphorylation under longer moxibustion, an additional group with 60 min moxibustion was performed following the procedure above.

2.4. Assays of Autophagy and Autophagic Pathways. A small number of fresh macrophages were fixed with $3 \%$ formaldehyde in phosphate buffer (PBS) for $10 \mathrm{~min}$ and permeabilized with $0.5 \%$ Triton X-100 in PBS for $10 \mathrm{~min}$. After washing with PBS twice, the cells were blocked with $1 \%$ bovine serum albumin in PBS with $0.1 \%$ Triton X-100 for $30 \mathrm{~min}$ and then incubated with rabbit polyclonal anti-LC3 antibody (1:200, MBL International, MA, USA) for $1 \mathrm{~h}$. After three washes with PBS with $0.1 \%$ Triton X-100, the cells were incubated with FITC-conjugated anti-rabbit IgG secondary antibody (1:500, Life Technologies) for $0.5 \mathrm{~h}$, counterstained with DAPI (4',6-diamidino-2-phenylindole, Sigma, St. Louis, $\mathrm{MO}$ ), and mounted in antifade agent (Invitrogen, Carlsbad, CA). Finally, LC3 staining was visualized by confocal fluorescence microscopy. The remaining macrophages were lysed and total protein extracts were subjected to western blots with anti-LC3 antibody, phospho-eIF2 $\alpha$ (Ser51) rabbit monoclonal antibody (mAb), phospho-Akt (Ser473) rabbit $\mathrm{mAb}$, and anti-Akt and eIF $2 \alpha$ rabbit antibodies $(1: 1000$, Cell Signaling Technology, MA, USA). Images were visualized by Licor Odyssey or Pierce ECL system.

2.5. Statistical Analysis. The survival rates were analyzed by a log-rank test; and the statistical analysis was performed with one-way analyses of variance (ANOVA).

\section{Results}

3.1. Moxibustion Promotes Autophagy in Macrophages. To assess whether moxibustion affected autophagy, moxibustion with a small size moxa stick was applied to the mouse abdomen Guanyuan point (CV4) for 15 and 30 minutes. Macrophages were then isolated from the peritoneal fluid after mice were sacrificed and subjected to autophagy assay. When macrophages were primed by starch and albumen, autophagic LC3-postive vacuoles were not observed in macrophages, similar to that seen in control mice, whereas LC3-positve puncta were greatly increased after $15 \mathrm{~min}$ or 30 min moxibustion (Figures 1(a) and 1(b)). Furthermore, when LC3 was detected in cell extracts, the ratio of LC3II was elevated following moxibustion compared with mock and primed conditions (Figure 1(c)). The levels of LC3-II protein and LC3 puncta were similar under both moxibustion conditions. These results suggest that moxibustion induces the macrophage autophagy in mice.

3.2. Moxibustion Induces eIF2 $\alpha$ Phosphorylation and Suppresses Akt Phosphorylation. We attempted to address upstream signaling pathways by which moxibustion activates autophagy. Akt inhibits endoplasmic reticulum (ER) stress by phosphorylating eIF2 $\alpha$-kinase PERK [35] and the PI3K/Akt/ mTOR pathway acts as a well-characterized negative regulator of autophagy [36]. Because moxibustion delivers heat stimulation to the operative site, we hypothesized it induced a stress response, and then Akt activity and ER stress were examined after moxibustion. To diminish the individual differences in mice, we investigated ER stress in mixed macrophages (Figure 2(a)) and random macrophages (Figure 2(b)). As shown in Figures 2(a) and 2(b) in top panel, phosphorylation of eIF $2 \alpha$ in macrophages was significantly elevated at $30 \mathrm{~min}$ and then slightly fell back at $1 \mathrm{~h}$ during moxibustion, indicating a rapid ER stress response was activated. Furthermore, the phosphorylation of Akt was decreased dramatically at both $30 \mathrm{~min}$ and $1 \mathrm{~h}$, suggesting moxibustion suppressed Akt activation (Figures 2(a) and 2(b) in bottom panel). Thus, we concluded that moxibustion induced autophagy in macrophages by suppressing the Akt pathway and activating ER stress. 

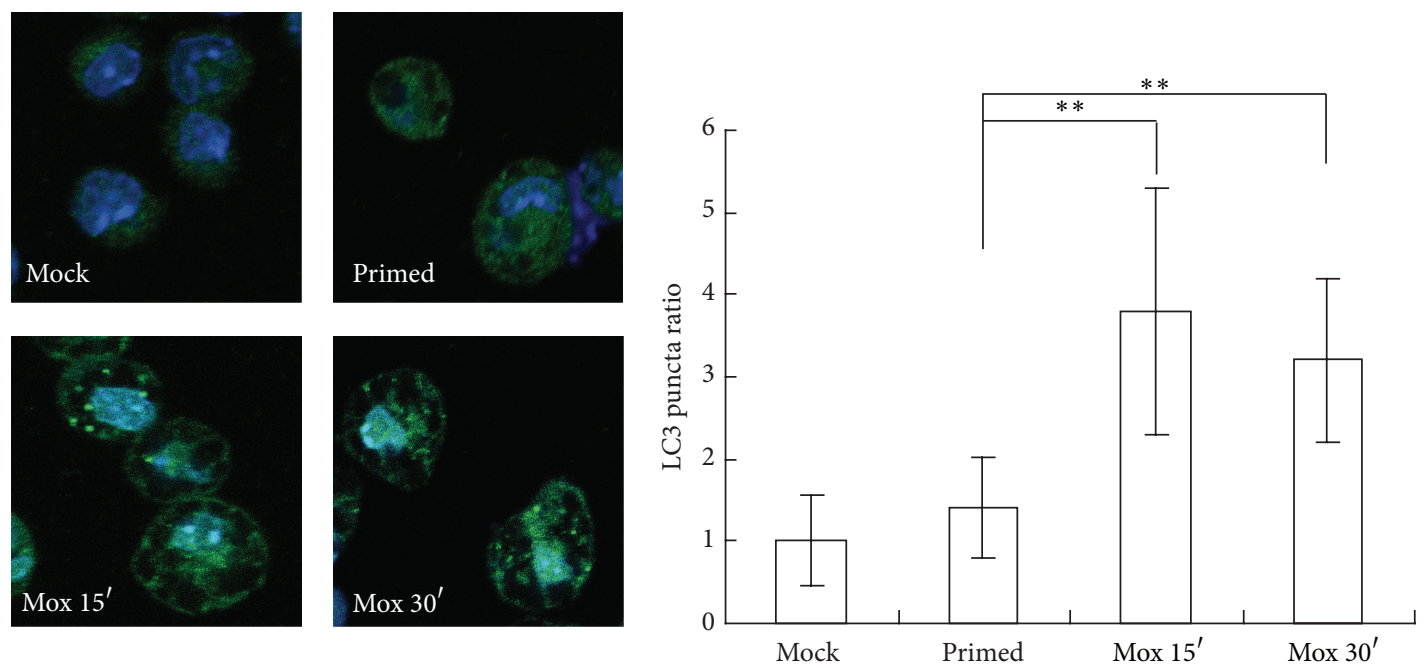

(a)

(b)

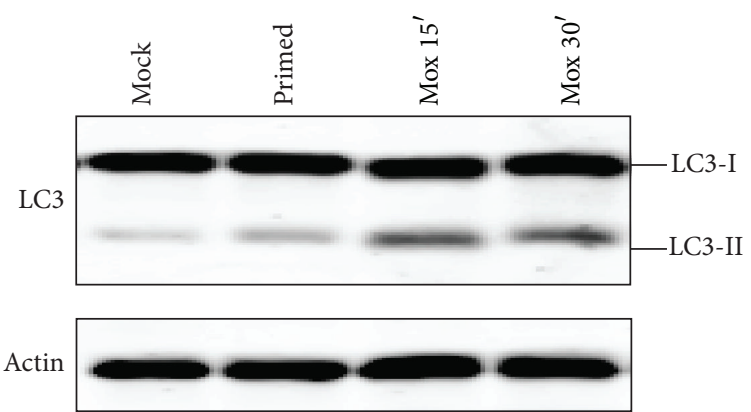

(c)

FIGURE 1: Autophagy is accelerated by moxibustion. Macrophages were collected from mice with or without moxibustion as indicated, fixed, and stained with anti-LC3 antibody, and LC3 staining was visualized by confocal fluorescence microscopy. Representative images (a) and the means of LC3 puncta/cell counted from 20 individual cells (b) are shown. ${ }^{* *} P<0.01$. Macrophages were lysed and equal mixtures of whole cell extracts were detected by western blots with anti-LC3 and anti-actin antibodies (c).

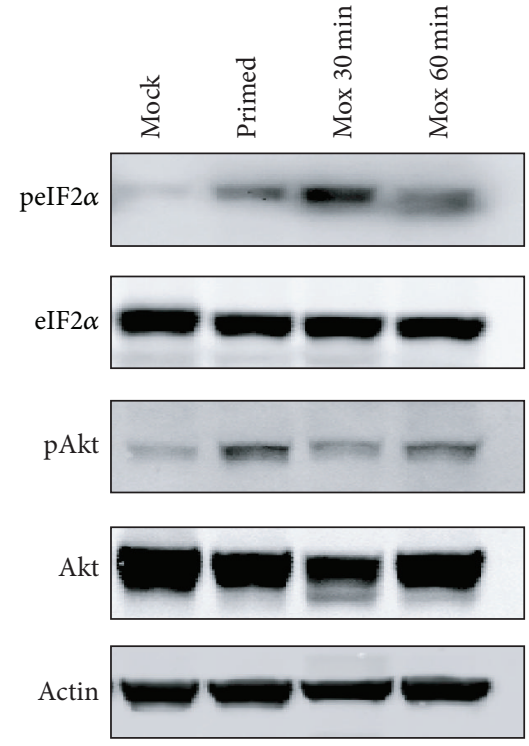

(a)
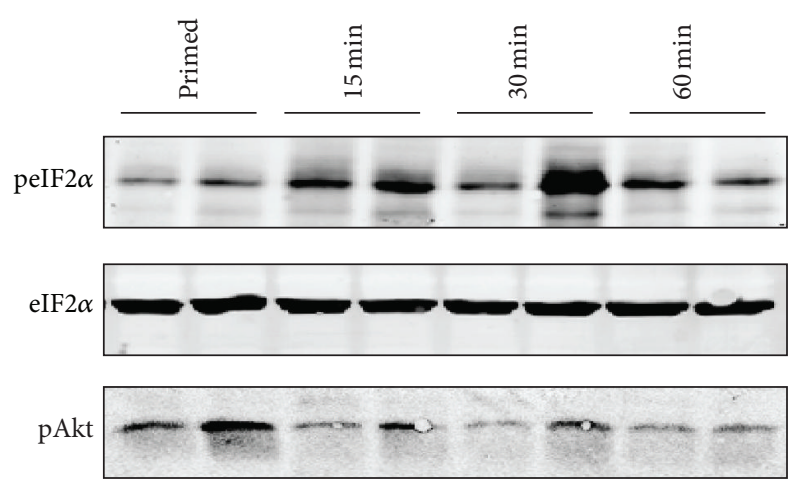

Akt

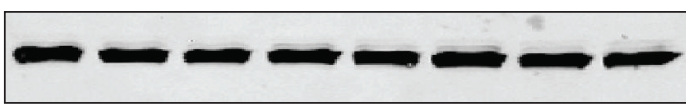

(b)

Figure 2: Autophagic Akt and eIF2 $\alpha$ pathways are activated by moxibustion. (a) Four mice per group were untreated or treated as indicated and equal whole cell extracts of macrophages from mice were mixed and signaling pathways detected as indicated. (b) Two random macrophage samples from six individual mice per group are shown. 


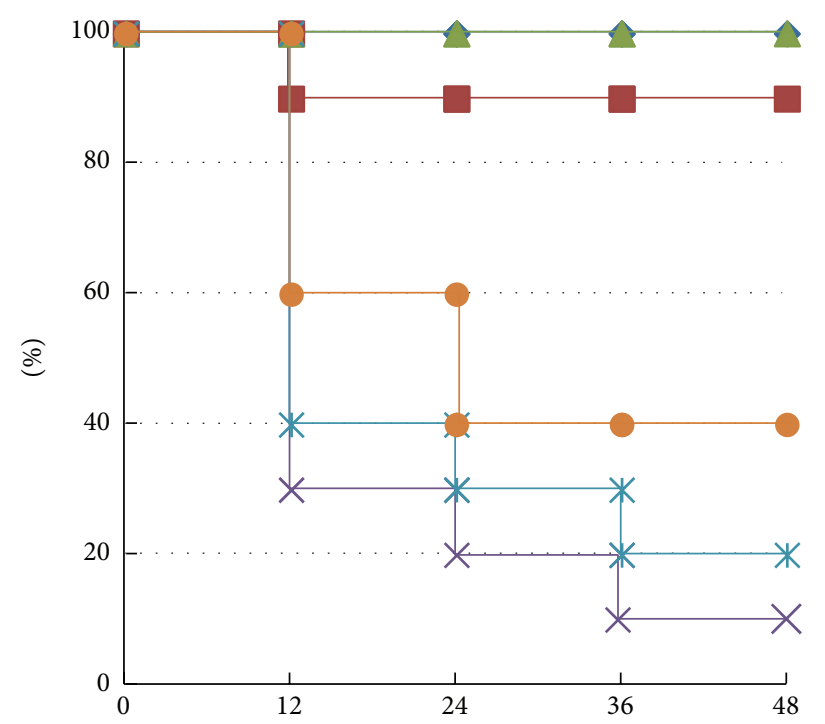

(h)

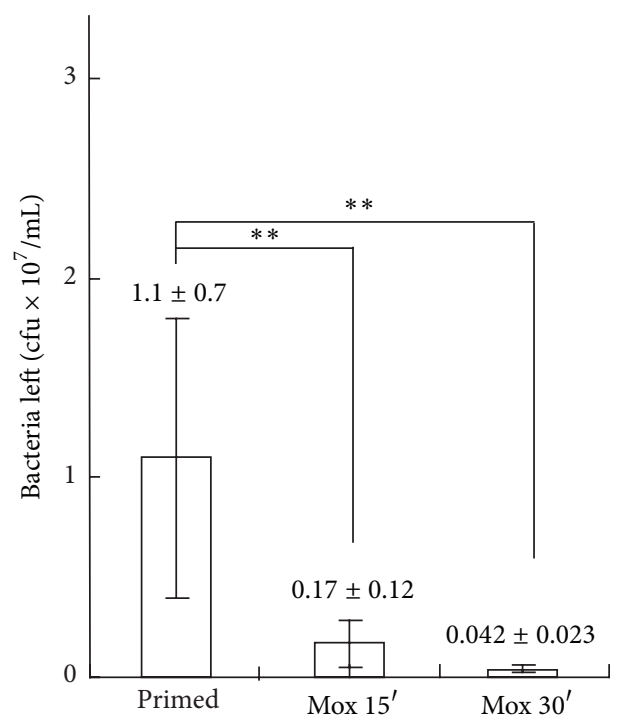

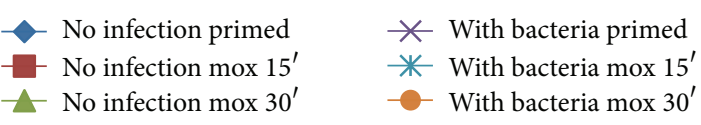

(a) (b)

FIgURE 3: Bacterial infection is alleviated by moxibustion. (a) Mice were injected intraperitoneally with $10 \mathrm{LD}_{50}$ Staphylococcus aureus as an acute bacterial model. At $1 \mathrm{~h}$ later, mice were untreated or administered moxibustion as indicated. The parallel tests without bacterial infection were performed following the same procedures. Survival was monitored for $48 \mathrm{~h}$ after moxibustion $(n=10 \mathrm{mice} / \mathrm{group})$ and the log-rank test was performed for the survival curve. $P<0.05$, primed group versus $30 \mathrm{~min}$ moxibustion group. (b) Once mice died or were sacrificed at $48 \mathrm{~h}$ after moxibustion, peritoneal fluids were collected immediately and plated on LB agar to measure the activity of bacterial colony formation. ${ }^{* *} P<0.01$.

3.3. Moxibustion Protects Experimental Mice from Bacterial Infection. Because elevated autophagy promotes bacterial clearance of macrophages, we hypothesized moxibustion would protect mice against bacterial infection with Staphylococcus aureus. Bacterial challenge caused $90 \%$ mortality in primed mice at $48 \mathrm{~h}$, during which the bacteria did not escape from killing by macrophages postinfection [37, 38]. The group receiving $15 \mathrm{~min}$ moxibustion showed little difference in survival compared with the control group, whereas the 30 min moxibustion group was significantly protected from death and acute infection (Figure 3(a)). This suggested the 30 min moxibustion induced a protective effect against bacterial infection. Furthermore, remaining bacteria were detected after moxibustion. As shown in Figure 3(b), the bacterial loads in the peritoneal fluid were dramatically reduced when mice were administered moxibustion for $15 \mathrm{~min}$ or $30 \mathrm{~min}$, although the latter was more effective. In conclusion, moxibustion provides a protective response and enhanced clearance of bacterial infection in macrophages.

\section{Discussion}

In the present study, we revealed that macrophage autophagy in the peritoneal fluid was elevated when moxibustion was applied to experimental mice. Moxibustion provided a protective response against bacterial infection. This effect probably contributes to the physiological stress response with Akt inhibition and activation of ER stress.

Moxibustion is one physical therapy in traditional medicine that utilizes heat stimulation to cure disease or quell pain. It might induce a series of signal pathways and stress through heat shock proteins [22, 39], including the Akt pathway and ER stress, as shown in the present study. Although the effect of moxibustion in the whole body requires more evidence, moxibustion is a suitable technique for local heat therapy to stimulate self-healing, recovery, and innate defense mechanisms. It is important to prevent severe heat stimuli by moxibustion that can cause burn injury following inflammation. However, heat therapy is beneficial when the heat stimulation is artificially controlled to a unique site without injury [40, 41].

Even though the effects of moxibustion have not yet been systemically evaluated, emerging studies show moxibustion can alter gene expression and signal transduction. The longdistance effect of moxibustion may be due to neural reflexes [25], whereas local effects are mediated through cytokines and signal transduction. The present study and previous studies have revealed moxibustion activates a unique cytokine profile and signal transduction pathways [22, 27-34, 42, 43], as well as elevating macrophage autophagy (Figure 1) and natural killer cell activity [34]. These results suggest moxibustion generates a protective response against viral 
and bacterial infection, although it is not clear whether it functions locally or systemically.

Although the studies have shown moxibustion contributes to the systemic and comprehensive immune, inflammatory, and analgesic activities, our finding represents a novel protective mechanism that the mild moxibustion promotes the autophagy and bactericidal function of macrophage. This effect is probably contributed to the inhibition of Akt phosphorylation and the activation of $\operatorname{eIF} 2 \alpha$ phosphorylation, two key signal pathways in a variety of stresses. However, the upstream signaling of moxibustion remains elusive and the further systematic analysis regarding metabolism and endocrine under moxibustion will greatly help to understand the mechanism. Alternatively, it is urgent to improve the clinical efficacy of moxibustion as well as to definete the indications and usage.

\section{Conclusion}

Our data provide evidence that moxibustion augments autophagy in macrophages and innate defense against bacterial infection. Based on our present study, mild moxibustion is effective at preventing and treating infectious disease in mice, acting as a simple and convenient activator of autophagy in physical therapy.

\section{Conflict of Interests}

The authors declare that there is no potential conflict of interests disclosed in this investigation.

\section{References}

[1] D. J. Klionsky, "Autophagy: from phenomenology to molecular understanding in less than a decade," Nature Reviews Molecular Cell Biology, vol. 8, no. 11, pp. 931-937, 2007.

[2] Z. Yang and D. J. Klionsky, "Mammalian autophagy: core molecular machinery and signaling regulation," Current Opinion in Cell Biology, vol. 22, no. 2, pp. 124-131, 2010.

[3] B. Levine, N. Mizushima, and H. W. Virgin, "Autophagy in immunity and inflammation," Nature, vol. 469, no. 7330, pp. 323-335, 2011.

[4] N. Mizushima and B. Levine, "Autophagy in mammalian development and differentiation," Nature Cell Biology, vol. 12, no. 9, pp. 823-830, 2010.

[5] A. M. Choi, S. W. Ryter, and B. Levine, "Autophagy in human health and disease," The New England Journal of Medicine, vol. 368, no. 7, pp. 651-662, 2013.

[6] B. Levine and G. Kroemer, "Autophagy in the pathogenesis of disease," Cell, vol. 132, no. 1, pp. 27-42, 2008.

[7] M. Kundu and C. B. Thompson, "Autophagy: basic principles and relevance to disease," Annual Review of Pathology: Mechanisms of Disease, vol. 3, pp. 427-455, 2008.

[8] D. C. Rubinsztein, P. Codogno, and B. Levine, "Autophagy modulation as a potential therapeutic target for diverse diseases," Nature Reviews Drug Discovery, vol. 11, no. 9, pp. 709-730, 2012.

[9] Z. J. Yang, C. E. Chee, S. Huang, and F. A. Sinicrope, “The role of autophagy in cancer: therapeutic implications," Molecular Cancer Therapeutics, vol. 10, no. 9, pp. 1533-1541, 2011.
[10] C. M. Kenific, A. Thorburn, and J. Debnath, "Autophagy and metastasis: another double-edged sword," Current Opinion in Cell Biology, vol. 22, no. 2, pp. 241-245, 2010.

[11] E. White and R. S. DiPaola, "The double-edged sword of autophagy modulation in cancer," Clinical Cancer Research, vol. 15, no. 17, pp. 5308-5316, 2009.

[12] T. Shintani and D. J. Klionsky, "Autophagy in health and disease: a double-edged sword," Science, vol. 306, no. 5698, pp. 990-995, 2004.

[13] J. Huang and J. H. Brumell, "Bacteria-autophagy interplay: a battle for survival," Nature Reviews Microbiology, vol. 12, no. 2, pp. 101-114, 2014.

[14] S. Mostowy, "Autophagy and bacterial clearance: a not so clear picture," Cellular Microbiology, vol. 15, no. 3, pp. 395-402, 2013.

[15] A. Choy and C. R. Roy, "Autophagy and bacterial infection: an evolving arms race," Trends in Microbiology, vol. 21, no. 9, pp. 451-456, 2013.

[16] J. Huang and J. H. Brumell, "Autophagy in immunity against intracellular bacteria," Current Topics in Microbiology and Immunology, vol. 335, no. 1, pp. 189-215, 2009.

[17] S. Shoji-Kawata, R. Sumpter, M. Leveno et al., "Identification of a candidate therapeutic autophagy-inducing peptide," Nature, vol. 494, no. 7436, pp. 201-206, 2013.

[18] E. Kuang, C. Y. M. Okumura, S. Sheffy-Levin et al., "Regulation of ATG4B stability by RNF5 limits basal levels of autophagy and influences susceptibility to bacterial infection," PLoS Genetics, vol. 8, no. 10, Article ID e1003007, 2012.

[19] S.-H. Yi, "Thermal properties of direct and indirect moxibustion," Journal of Acupuncture and Meridian Studies, vol. 2, no. 4, pp. 273-279, 2009.

[20] S. Y. Kim, S. H. Yi, J. H. Cho, C. S. Yin, H. Lee, and H. J. Park, "Heat stimulation on the skin for medical treatment: can it be controlled?" Journal of Alternative and Complementary Medicine, vol. 17, no. 6, pp. 497-504, 2011.

[21] J. H. Chiu, "How does moxibustion possibly work?” EvidenceBased Complementary and Alternative Medicine, vol. 2013, Article ID 198584, 8 pages, 2013.

[22] Y. O. Cakmak, "A review of the potential effect of electroacupuncture and moxibustion on cell repair and survival: the role of heat shock proteins," Acupuncture in Medicine, vol. 27, no. 4, pp. 183-186, 2009.

[23] H. Deng and X. Shen, "The mechanism of moxibustion: ancient theory and modern research," Evidence-Based Complementary and Alternative Medicine, vol. 2013, Article ID 379291, 7 pages, 2013.

[24] I. Jang and J. Park, "Can carbon dioxide laser substitute moxibustion?" Lasers in Medical Science, vol. 24, no. 2, p. 290, 2009.

[25] E. Noguchi, H. Ohsawa, and K. Takagi, "Neural mechanism of localized changes in skeletal muscle blood flow caused by moxibustion-like thermal stimulation of anesthetized rats," Journal of Physiological Sciences, vol. 59, no. 6, pp. 421-427, 2009.

[26] X. Shen, L. Zhao, G. Ding et al., "Effect of combined laser acupuncture on knee osteoarthritis: a pilot study," Lasers in Medical Science, vol. 24, no. 2, pp. 129-136, 2009.

[27] E. Zhou, H. Liu, H. Wu et al., "Down-regulation of protein and mRNA expression of IL-8 and ICAM-1 in colon tissue of ulcerative colitis patients by partition-herb moxibustion," Digestive Diseases and Sciences, vol. 54, no. 10, pp. 2198-2206, 2009. 
[28] C. Bao, L. Wu, H. Wu et al., "Moxibustion inhibits apoptosis and tumor necrosis factor-alpha/tumor necrosis factor receptor 1 in the colonic epithelium of crohn's disease model rats," Digestive Diseases and Sciences, vol. 57, no. 9, pp. 2286-2295, 2012.

[29] M. Kogure, N. Mimura, H. Ikemoto et al., "Moxibustion at mingmen reduces inflammation and decreases IL-6 in a collagen-induced arthritis mouse model," Journal of Acupuncture and Meridian Studies, vol. 5, no. 1, pp. 29-33, 2012.

[30] X. M. Wang, Y. Lu, L. Y. Wu et al., "Moxibustion inhibits interleukin-12 and tumor necrosis factor alpha and modulates intestinal flora in rat with ulcerative colitis," World Journal of Gastroenterology, vol. 18, no. 46, pp. 6819-6828, 2012.

[31] Y. Shi, Y. Cui, H. Wu et al., "Effects of mild-warming moxibustion on Bcl-2 and PKC expressions of peripheral blood in elderly people," Journal of Traditional Chinese Medicine, vol. 32, no. 1, pp. 45-51, 2012.

[32] Z. H. Yue, X. Q. He, X. R. Chang et al., "The effect of herbpartition moxibustion on toll-like receptor 4 in rabbit aorta during atherosclerosis," Journal of Acupuncture and Meridian Studies, vol. 5, no. 2, pp. 72-79, 2012.

[33] X. Wang, Y. Lu, L. Wu et al., "Moxibustion inhibits the ERK signaling pathway and intestinal fibrosis in rats with Crohn's disease," Evidence-Based Complementary and Alternative Medicine, vol. 2013, Article ID 198282, 12 pages, 2013.

[34] Y. Takayama, M. Itoi, T. Hamahashi et al., "Moxibustion activates host defense against herpes simplex virus type I through augmentation of cytokine production," Microbiology and Immunology, vol. 54, no. 9, pp. 551-557, 2010.

[35] Z. Mounir, J. L. Krishnamoorthy, S. Wang et al., "Akt determines cell fate through inhibition of the PERK-eIF2 $\alpha$ phosphorylation pathway," Science Signaling, vol. 4, no. 192, article ra62, 2011.

[36] G. Kroemer, G. Mariño, and B. Levine, "Autophagy and the integrated stress response," Molecular Cell, vol. 40, no. 2, pp. 280-293, 2010.

[37] M. Kubica, K. Guzik, J. Koziel et al., "A potential new pathway for Staphylococcus aureus dissemination: the silent survival of S. aureus phagocytosed by human monocyte-derived macrophages," PLoS ONE, vol. 3, no. 1, Article ID e1409, 2008.

[38] G. J. Jakab and G. M. Green, "Defect in intracellular killing of Staphylococcus aureus within alveolar macrophages in Sendai virus infected murine lungs," Journal of Clinical Investigation, vol. 57, no. 6, pp. 1533-1539, 1976.

[39] J. Yu, H. Peng, Y. Lin, and S. Yi, "Effect of moxibustion treatment on cell apoptosis and expressions of heat shock protein and second mitochondrial activator of caspase in acute gastric mucosal lesion of rats," Journal of Traditional Chinese Medicine, vol. 33, no. 2, pp. 258-261, 2013.

[40] S. I. Zeitlin, "Heat therapy in the treatment of prostatitis," Urology, vol. 60, supplement 6, pp. 38-41, 2002.

[41] A. Chandler, J. Preece, and S. Lister, "Using heat therapy for pain management," Nursing Standard, vol. 17, no. 9, pp. 40-42, 2002.

[42] K. Kimura, H. Takeuchi, K. Yuri, and I. Wakayama, "Inhibition of nitric oxide synthase attenuates cutaneous vasodilation during warm moxibustion-like thermal stimulation in humans," Journal of Alternative and Complementary Medicine, vol. 18, no. 10, pp. 965-970, 2012.

[43] H. Y. Yin, Y. Tang, S. F. Lu et al., "Gene expression profiles at moxibustioned site (ST36): a microarray analysis," EvidenceBased Complementary and Alternative Medicine, vol. 2013, Article ID 890579, 7 pages, 2013. 


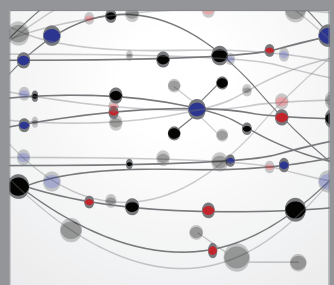

The Scientific World Journal
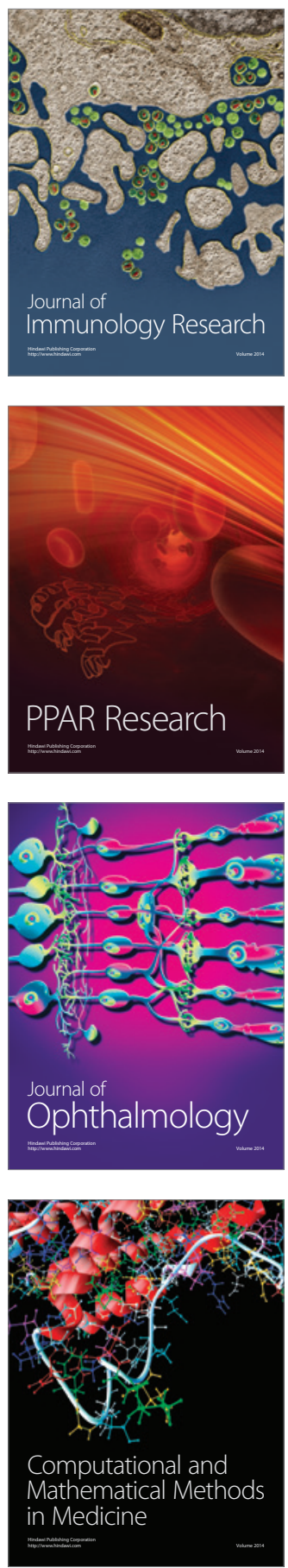

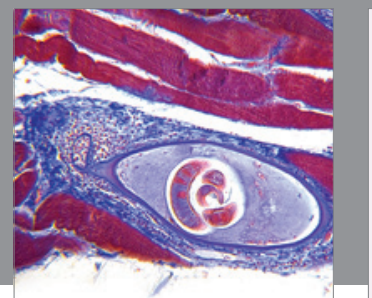

Gastroenterology

Research and Practice
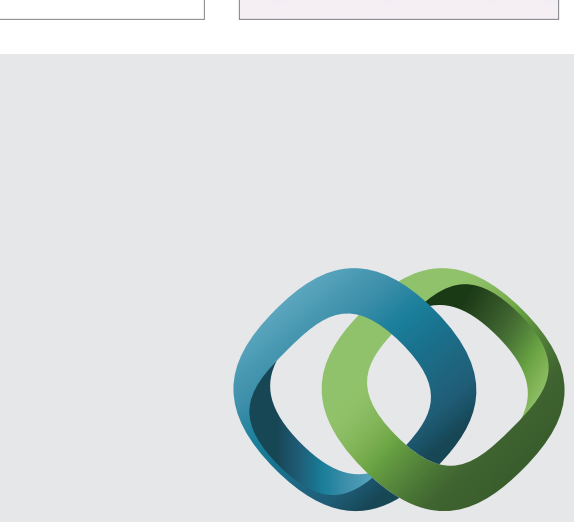

\section{Hindawi}

Submit your manuscripts at

http://www.hindawi.com
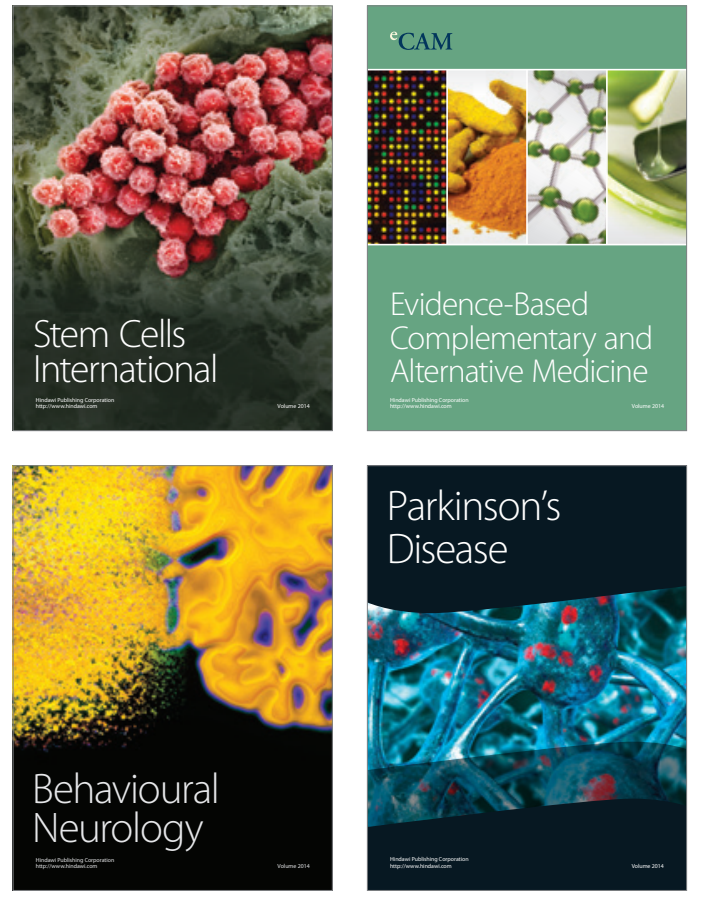
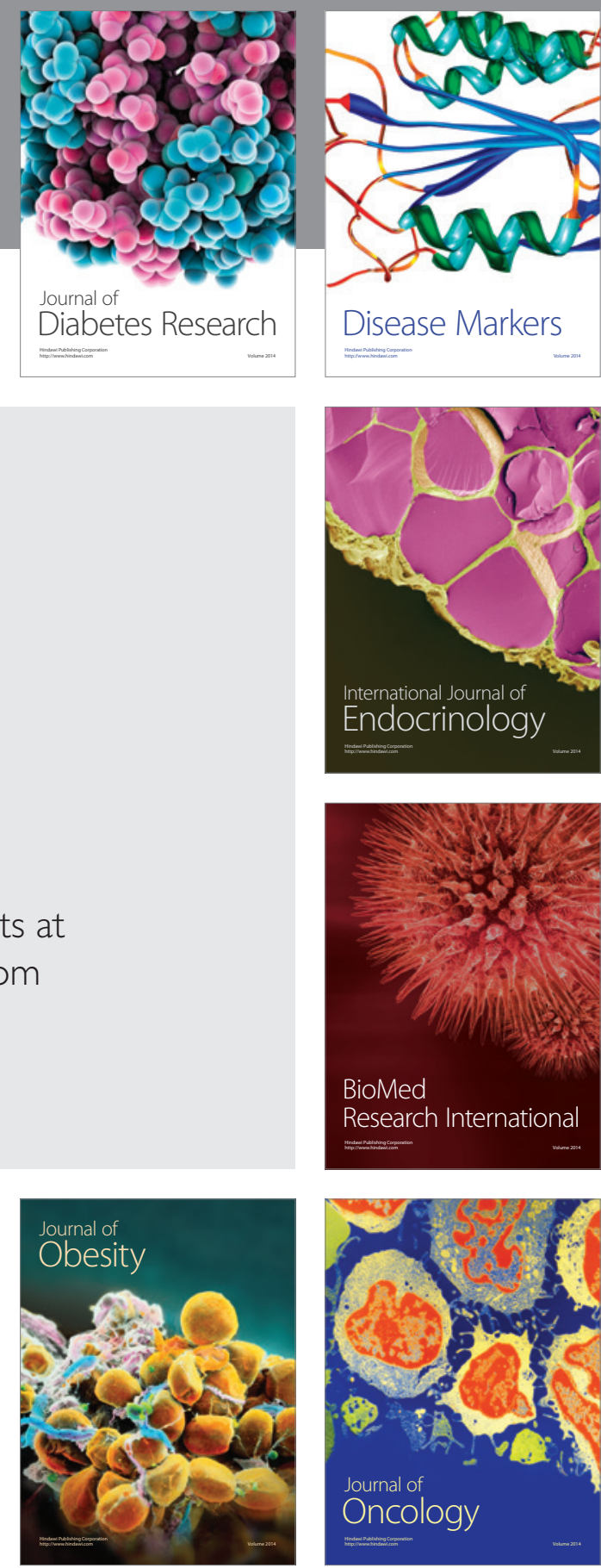

Disease Markers
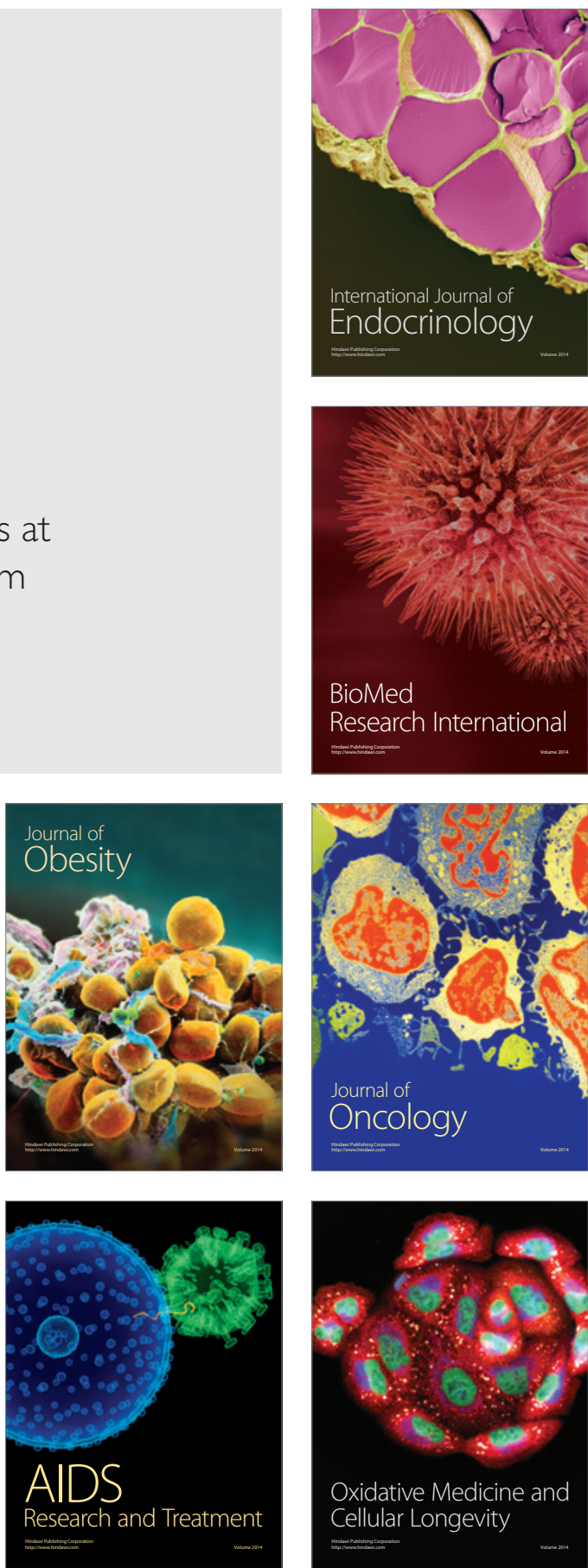